Research Paper

\title{
High Expression of Pyruvate Kinase M2 is Associated with Chemosensitivity to Epirubicin and 5-Fluorouracil in Breast Cancer
}

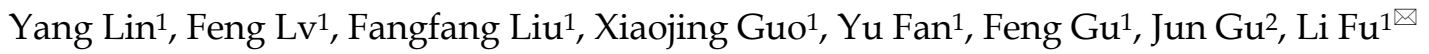 \\ 1. Department of Breast Cancer Pathology and Research Laboratory, Key Laboratory of Breast Cancer Prevention and Therapy (Ministry of Educa- \\ tion), National Clinical Research Center for Cancer, Tianjin Medical University Cancer Institute and Hospital, National Clinical Research Center \\ of Cancer, Tianjin, 300060, China \\ 2. Wadsworth Center, New York State Department of Health, and School of Public Health, State University of New York at Albany, NY 12201, \\ United States
}

$\triangle$ Corresponding author: Li Fu, M.D, Ph.D, Director, Department of Breast Pathology and Research Lab, Tianjin Medical University Cancer Institute and Hospital, Huanhuxi Road, Hexi District, Tianjin, China, 300060. Tel: 86-22-2334-0123-6118; Fax: 86-22-2334-0123-6118; E-mail: fuli@tijmu.edu.cn

() 2015 Ivyspring International Publisher. Reproduction is permitted for personal, noncommercial use, provided that the article is in whole, unmodified, and properly cited. See http:/ /ivyspring.com/terms for terms and conditions.

Received: 2015.05.16; Accepted: 2015.07.30; Published: 2015.09.15

\begin{abstract}
Background: Pyruvate kinase M2 (PKM2) is the key enzyme in the Warburg effect, and it was recently reported to be involved in the metabolic pathways of chemotherapeutic drugs. However, the role of PKM2 in breast cancer and its influence in the sensitivity to front-line anticancer drugs remains unclear.

Methods: In this study, we examined the correlation between the expression of PKM2 and the sensitivity of primary breast cancer cells to anticancer drugs. PKM2 expression was studied by immunohistochemistry using biopsy samples of 296 patients diagnosed with invasive breast carcinoma, and the collagen gel droplet embedded culture-drug sensitivity tests (CD-DST) was conducted to all the patients to detect in vitro chemosensitivity after surgery.

Results: We found high PKM2 expression was significantly associated with in vitro chemosensitivity to epirubicin $(\mathrm{EPI})(\mathrm{P}=0.019)$ and 5 -fluorouracil $(5-\mathrm{Fu})(\mathrm{P}=0.009)$ in breast cancer patients. Then we used a small group of neoadjuvant chemotherapy cases to confirm that the higher PKM2 expression, the better pathological response to therapy was obtained in patients treated with EPI-based or EPI plus 5-Fu chemotherapy regimens. Although univariate and multivariate analysis indicated that high PKM2 was a poor independent predictor of progression free survival (PFS) and overall survival (OS) in breast cancer, patients with PKM2 high expression who received EPI-based or EPI plus 5-Fu chemotherapy were found to have a longer PFS $(P=0.003, P=0.013)$ and OS $(P=0.003, P=0.004)$ than patients treated with non-EPI/5-Fu-based regimens, respectively.

Conclusions: Our findings confirmed the poor prognosis of high PKM2 expression in breast cancer patients and revealed the predictive value of high PKM2 in the therapeutic response to EPI and 5-Fu. Moreover, our results provide the guidance of individual treatment for breast cancer patients who are foreboded a poor prognosis by the presence of high PKM2 status.
\end{abstract}

Key words: Pyruvate kinase M2, breast cancer chemosensitivity, epirubicin, 5-fluorouracil

\section{Introduction}

Breast cancer, the highest incidence in cancers, is the principal cause of cancer death in females' worldwide [1]. Failure of postoperative adjuvant chemotherapy is the most vital reason of breast cancer recurrence and metastasis. There is a demand to accurately determine patients' individual sensitivity to chemotherapy drugs to improve their chemotherapeutic efficacy. 
CD-DST is a reliable emerging chemosensitivity test which having many benefits for clinical usefulness compared with conventional tests [2]. With requiring a small number of cells and maintaining the original growth characteristics, CD-DST shows a favorable successful rate in primary cells culture. By applying physiological concentrations of anticancer drugs and analyzing results through image analysis system, CD-DST could predict the therapeutic response to anticancer drugs for patients. Previous studies have already claimed CD-DST could be helpful for planning optimal chemotherapy schemes for gastric cancer [2], ovarian cancer [3], breast cancer [4] and colorectal cancer [5], with an intense correlation between clinical prognosis and CD-DST results being found [2-5]. However, CD-DST requires a long time to perform, and it is very costly for patients.

Regulation of cell metabolism is a leading characteristic of many cancer cells in tumor physiology [6]. Cancer cells obtain most of their energy by glycolysis even in the existence of adequate oxygen, which was called the "Warburg effect" [7]. PKM2 is the pivotal metabolic molecule in the process of Warburg effect $[8,9]$ and catalyzes the final step of aerobic glycolysis [10]. In tumor microenvironment, PKM2 expressed in malignant cells and maintained their glycolytic phenotype $[11,12]$. Some recent studies have already related PKM2 status with cancer cells' response to chemotherapy and radiotherapy [13, 14]. There are few published reports which have described the biological role of PKM2 in clinical breast cancer patients; moreover, its influence on chemosensitivity to front-line anticancer drugs of breast cancer is still unclear.

In the current research, we explored the correlation between PKM2 expression and the patients' primary tumors CD-DST results to evaluate the influence of PKM2 levels in individual chemosensitivity to breast cancer. We then used a small number of neoadjuvant chemotherapy cases to verify the conclusion that the higher PKM2 status was related to breast cancer patients' chemosensitivity to EPI and 5-Fu. Furthermore, we adopted the survival analysis to assess the long-term effect of PKM2 status to the efficacy of EPI and 5-Fu in breast cancer patients. Our findings revealed the predictive value of PKM2 in therapeutic response to EPI-based or EPI plus 5-Fu chemotherapy in breast cancer. Moreover, our results suggested that evaluating tumors' PKM2 status provide a new option to optimize individual chemotherapy regimens.

\section{Materials and Methods}

\section{Patient selection and clinical information}

Paraffin-embedded tumor tissues were analyzed from 296 patients (mean age: 51 years, range from 23 to 83 years) which were confirmed between January 2008 and December 2009. Patients' information was recorded in the files of Cancer Hospital, Tianjin Medical University. All the patients had been diagnosed as invasive breast carcinoma by preoperative needle core biopsy or intraoperative frozen section examination. Two hundred and ninety-two patients had radical mastectomy or modified radical mastectomy and four patients had lumpectomy after diagnosis. CD-DST was conducted by using surgically resected fresh specimens. No patients had received chemotherapy prior to hospitalization. There were 5 patients with distant metastasis at initial diagnosis. All the histopathological sections were reviewed and confirmed diagnosis by three pathologists. Histologic types were determined according to the World Health Organization (WHO) classification. There were 293 (99\%) cases of invasive ductal carcinoma, not otherwise specified type (NOS-IDC) and 3 (1\%) cases of invasive lobular carcinoma. Histologic grading was identified by the modified Bloom-Richardson grading system [15]. Two hundred and seventy-four (92.6\%) cases were belong to histological grade II - III. Patient's consent for research was obtained prior to surgery and the research was given official approval by the Institutional Research and Ethical Committee of Tianjin Medical University. The follow-up visit was from 2 to 82 months (median time: 52 months), during the time $53(17.9 \%)$ patients suffered local tumor recurrence or distant metastasis, and 43(14.5\%) patients died owing to cancer.

\section{Drugs sensitivity test by CD-DST}

According to the previously reported method $[16,17]$, the CD-DST kit (Nitta Gelatin, Japan) was applied to perform in vitro chemosensitivity test. Fresh specimens from surgical excision of the tumor were suspended and digested in the cell dispersion enzyme (Nitta Gelatin, Japan) which diluted by Hanks' balanced saline solution (HBSS). Following removing all dead cells, living cells were gathered and incubated in collagen gel droplet embedded culture $\left(3 \times 10^{2}\right.$ cells per collagen gel droplet) at $37^{\circ} \mathrm{C}$ overnight. Then cells were treated with Epirubicin (EPI; $0.3 \mu \mathrm{g} / \mathrm{ml}$ ), Cisplatin (CDDP; $2 \mu \mathrm{g} / \mathrm{ml}$ ), Vinorelbine (NVB; $10 \mu \mathrm{g} / \mathrm{ml})$, Paclitaxel (PAC; $10 \mu \mathrm{g} / \mathrm{ml}$ ), 5-Fluorouracil $(5-\mathrm{Fu} ; 10 \mu \mathrm{g} / \mathrm{ml})$, and each patient received 4 types of anticancer drugs randomly. The final drugs' concentrations were comparable to the standard levels in vivo. $\mathrm{A} \mathrm{CO}_{2}$ incubator was used to incubate the cells at $37^{\circ} \mathrm{C}$ for $24 \mathrm{~h}$. Following clearing away the drugs included medium, cells were incubated in PCM-2 medium (Kurabo, Japan) for 7 days. The medium was changed once on the 4 th day of ex- 
periment. The colonies in the collagen gel droplets were stained by Neutral red, and then fixed in $10 \%$ formalin.

The sensitivity of the tumor cells in vitro was denoted by the percentage of $\mathrm{T} / \mathrm{C}$ ratio. $\mathrm{T}$ indicated the total quantity of living cells in the experimental group, while $C$ stood for the total amount of living cells in the control group; when the $\mathrm{T} / \mathrm{C}$ ratio $\leq 50 \%$, it was deemed to represent in vitro sensitivity; when the $\mathrm{T} / \mathrm{C}$ ratio $>50 \%$, it was considered to denote non-sensitivity. By applying the cut-off ratio at $50 \%$, patients were separated into chemotherapy sensitive group and non-sensitive group [18].

\section{PKM2 immunohistochemistry and quantifica- tion}

Paraffin-embedded tissue sections of 296 invasive breast tumors were immunostained using an automated staining platform (Dako Autostainer Link48+PT Link, DakoCytomation, Glostrup, Denmark). Tissue sections were pretreated with Dako PT LINK which containing Target Retrieval solution ( $\mathrm{pH}$ 9, S1700, Dako, Inc.) at $97^{\circ} \mathrm{C}$ for $20 \mathrm{~min}$, followed by endogenous enzyme block at room temperature for 5 min, then incubated with 1:800 diluted PKM2 rabbit monoclonal antibody (D78A4,Cell Signaling Technology, Inc.) at room temperature for $25 \mathrm{~min}$. The UltraVision $^{\mathrm{TM}}$ Quanto Detection System HRP DAB kit (Thermo Scientific, Inc.) was followed by standard signal amplification including Poxt primary block (Lot.O2Q140731) at room temperature for $10 \mathrm{~min}$, HRP polymer (Lot.QHL140804) at $25^{\circ} \mathrm{C}$ for $15 \mathrm{~min}$, DAB reaction (Lot.QNDX140805) for $6 \mathrm{~min}$ and hematoxylin staining for $3 \mathrm{~min}$. The staining was evaluated by $\mathrm{H}$ score immunohistochemical assessment. $\mathrm{H}$-score assessment was due to the staining intensity (0-3) (0, denoting negative; $1+$, denoting weak; $2+$, denoting moderate; and $3+$, denoting strong) and the percentage of positive cells $(0-100 \%)$. Each sample's intensity level was multiplied by the percentage of positive cells, and the final $\mathrm{H}$ score was acquired by adding all the values, ranging from 0 to 300 . $\mathrm{H}$-score of PKM2 IHC ranged from 0 to 285 (Fig.1), with the median value of 90 was the cutoff value for separating PKM2 high expression group from PKM2 low expression group.

\section{Immunohistochemistry for molecular sub- types}

Serial paraffin-embedded tissue sections were used to perform additional immunohistochemistry staining for molecular subtypes by using the standard procedures of our lab. Primary antibodies were adopted as follows in the light of manufacturer's instructions: ER (1:150, Zymed, CA), PR (1:150, Zymed),
HER2 (DAKO HercepTestTM, Denmark), Ki-67 (1:200, ThermoScientific, CA), EGFR (1:100, Zymed), and CK5/6 (1:100, Zymed). We adopt the method of Cheang et al [19] reported to evaluate the staining of these biomarkers. 2+HER2 expression tumors were verified by fluorescence in situ hybridization (FISH). Breast cancer molecular subtypes were defined as follows: luminal A (ER positive and/or PR positive as well as Ki-67 < $20 \%$ ), luminal B (ER positive and/or PR positive as well as Ki-67 $\geq 20 \%$; or ER positive and/or PR positive and HER2 positive), HER2 enriched (ER and PR both negative while HER2 positive), and basal-like (ER, PR and HER2 all negative, while CK5/6 and/or EGFR positive) [20].

\section{Neoadjuvant chemotherapy verification cases}

To confirm the correlation between PKM2 expression and chemosensitity to EPI and 5-Fu, 23 patients received EPI-based (without 5-Fu) neoadjuvant chemotherapy and 25 patients received EPI plus 5-Fu based neoadjuvant chemotherapy were adopted to perform a small samples validation test. Regimens containing $5-\mathrm{Fu}$ without EPI are rare, because 5-Fu always combines with EPI in breast cancer chemotherapy schemes. To investigate the correlation between PKM2 and chemosensitivity to 5-Fu, the regimens we used which included both EPI and 5-Fu. All patients were confirmed as breast invasive carcinoma by needle core biopsy between January 2009 and December 2009 in Cancer Hospital, Tianjin Medical University. All the patients completed a full protocol (4 cycles) of neoadjuvant chemotherapy. PKM2 was detected by using needle core biopsy before chemotherapy. Tumor's response to neoadjuvant chemotherapy was assessed histologically on excised specimens using a previously modified method [21]. None of invasive cancer in breast and axillary lymph nodes represented pathological complete response (pCR). Existence of less than 10 microscopic foci of invasive cancer cells in breast and/or axillary lymph nodes stood for pathological partial response (pPR). Otherwise, patients were considered to have no pathologic response ( $\mathrm{pNR}$ ).

\section{Statistical analysis}

Statistical analyses were conducted using SPSS 16.0 software package. Independent samples T-test was used to compare the differences between groups. The association between patients' characteristics and PKM2 expression was evaluated by Chi-Square test or Fisher's exact test. The Kaplan-Meier method was used to estimate progression free survival (PFS) and overall survival (OS), and the log-rank test was conducted to compare survival differences between groups. Univariate and multivariate analysis was on 
the basis of the Cox proportional hazards regression model. A two-sided $P$ value of $<0.05$ was considered statistically significant in all the analyses.

\section{Results}

\section{Correlation of PKM2 expression with Patient characteristics and Clinicopathological fea- tures}

PKM2 was assessed using IHC in 296 invasive breast cancer cases (Fig.1) which received CD-DST. There were 143(48.3\%) cases in the PKM2 low expression group and $153(51.7 \%)$ cases in the PKM2 high expression group. The information of PKM2 expression with patients' characteristics and clinicopathological features were summarized in Table 1 . In these 296 invasive breast cancer cases, PKM2 was negatively correlated with tumor size $(\mathrm{P}=0.008)$, while positively correlated with lymph node stage $(\mathrm{P}=0.047)$. No significant differences in patients' age, menopausal status, family history of cancer, histological grade, the status of ER/PR/HER2/Ki67, molecular subtypes and clinical staging were obtained $(\mathrm{P}>0.05)$.

\section{PKM2 expression is associated with tumor cells chemosensitivity to EPI and 5 -Fu in vitro}

Patients' CD-DST results were displayed in Table 2. The PKM2 expression score was significantly higher in EPI sensitive group compared to EPI non-sensitive group ( $\mathrm{P}=0.019)$ (Fig.2A), while this difference of PKM2 expression was more significant between 5-Fu sensitive group and 5-Fu non-sensitive group ( $\mathrm{P}=0.009)$ (Fig.2E). No significant differences in PKM2 expression were obtained between the sensitive and non-sensitive groups of CDDP, NVB, and PAC (P >0.05) (Fig.2B, 2C and 2D).

\section{Neoadjuvant chemotherapy cases confirmed the association between PKM2 expression and $\mathrm{EPI} / 5-\mathrm{Fu}$ chemosensitivity in vivo}

In order to explore if PKM2 status was correlated with chemosensitivity to EPI and 5-Fu in vivo, we included a subgroup of patients who received neoadjuvant chemotherapy prior to surgery. PKM2 was detected by using needle core biopsy before chemotherapy. Pathological response to therapy was measured in all patients. In the group of EPI-based (without 5-Fu) neoadjuvant chemotherapy, the expression of PKM2 in patients who received pCR was significantly higher than those who did not obtain apparently pathological response (pNR) ( $\mathrm{P}=0.004)$ (Fig.3A). At the same time, in the group of EPI plus 5-Fu neoadjuvant chemotherapy, the expression of PKM2 was shown to be significantly higher in both $\mathrm{pCR}$ and $\mathrm{pPR}$ cases than in pNR cases $(\mathrm{P}=0.000, \mathrm{P}=0.042)$. In addition, there was also a significant difference of PKM2 expression between $\mathrm{pCR}$ and $\mathrm{pPR}$ cases in this group $(\mathrm{P}=0.039)$. (Fig.3B)
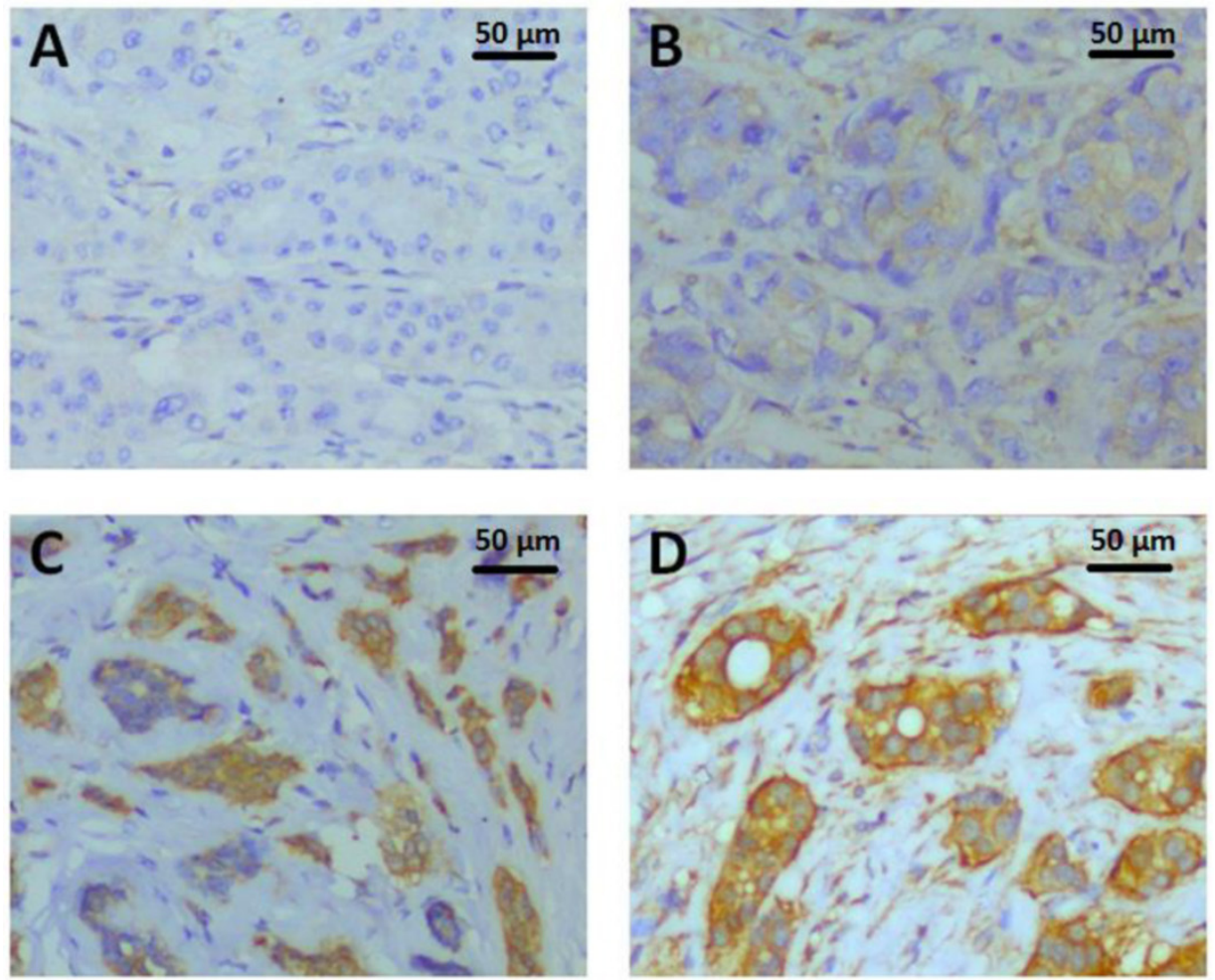

Fig 1. PKM2 expression detected by immunohistochemistry. The representative PKM2 staining intensities were localized primarily in the cytoplasm of breast cancer cells. A. Intensity 0, negative; B. Intensity 1+, weak; C. Intensity 2+, moderate; D. Intensity 3+, strong; 
A.

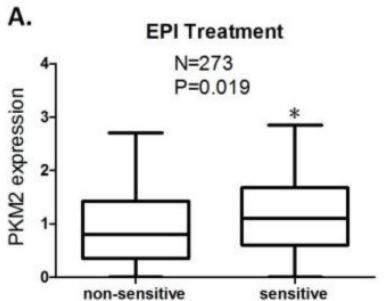

D.

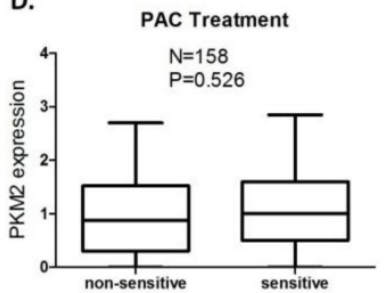

B.
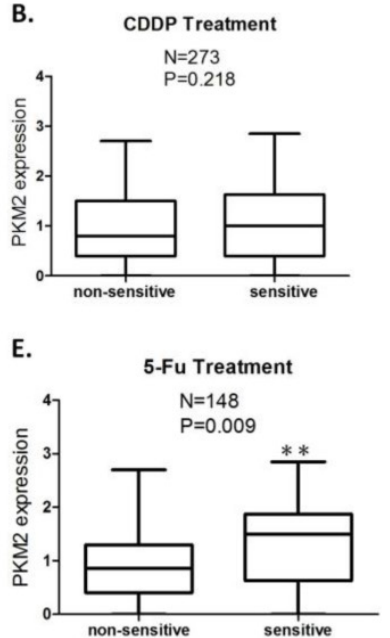

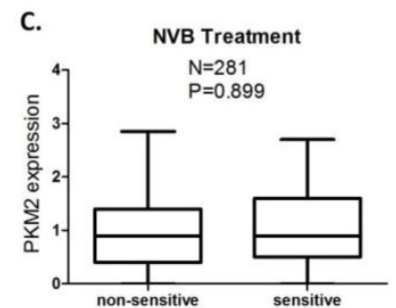

Fig 2. PKM2 expression status in sensitive and non-sensitive groups of each anticancer drug. The PKM2 expression score was significantly higher in the group sensitive to $\mathrm{EPI}(\mathrm{P}=0.019)(\mathrm{A})$ and $5-\mathrm{Fu}(\mathrm{P}=0.009)(\mathrm{E})$ compared to the group non-sensitive to $\mathrm{EPI}$ and 5 -Fu, respectively; No significant differences in $\mathrm{PKM} 2$ expression were obtained between sensitive and non-sensitive groups of CDDP (B), NVB (C), and PAC (D). Analysis was limited to patients for whom data were available; Vertical axis coordinate: $1=100 \mathrm{H}$-score; $* \mathrm{P}<0.05$, $* * \mathrm{P}<0.01$.
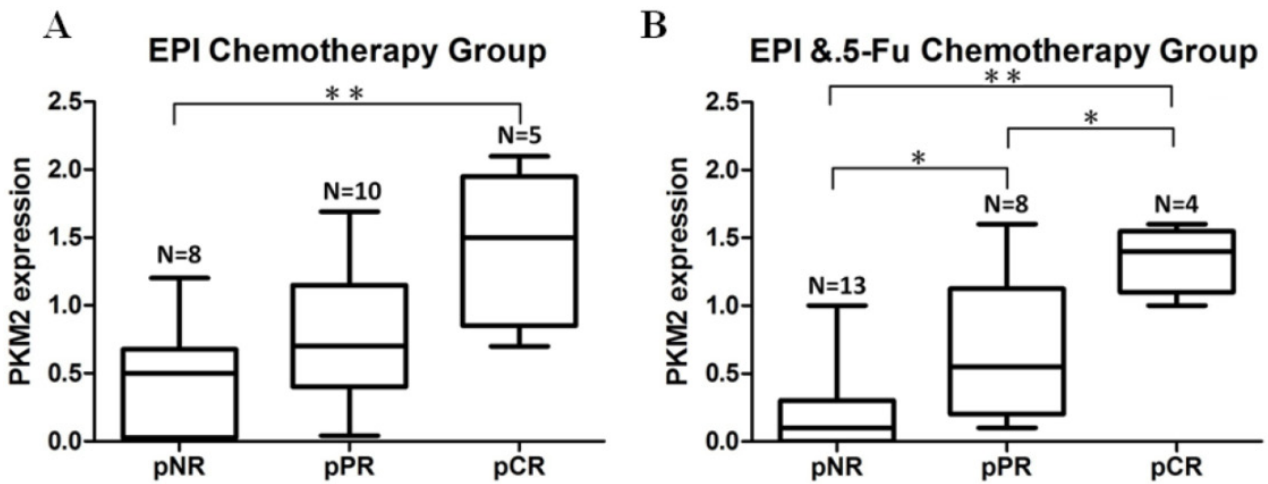

Fig 3. Neoadjuvant chemotherapy cases confirmed the association between PKM2 expression and EPI/5-Fu chemosensitivity. A. EPI-based (without 5-Fu) neoadjuvant chemotherapy group $(N=23)$; $B$. EPI plus 5-Fu neoadjuvant chemotherapy group $(N=25)$. pNR, no pathological response; $p P R$, pathological partial response; $P C R$, pathological complete response. $* \mathrm{P}<0.05$, $* * \mathrm{P}<0.01$

\section{PKM2 expression and the survival of breast cancer patients}

The Kaplan-Meier estimator and the log rank test revealed that high expression of PKM2 was indicated to be an unfavorable indicator evaluated by PFS $\left(\chi^{2}=8.712, P=0.003\right)$ (Fig.4A) and OS $\left(\chi^{2}=7.145, P=\right.$ 0.008 ) (Fig.4B) compared with low PKM2 expression.

The univariate analysis indicated that tumor size, lymph node status, HER2 status, Ki67 status and PKM2 status were significantly correlated with poor PFS and OS $(\mathrm{P}<0.05)$ (Table 3). In multivariate Cox proportional hazards model, lymph node status (PFS, $\mathrm{P}=0.045 ; \mathrm{OS}, \mathrm{P}=0.029)$, Ki67 status (PFS, $\mathrm{P}=0.007$; OS, $\mathrm{P}=0.002$ ) and $\mathrm{PKM} 2$ status (PFS, $\mathrm{P}=0.015 ; \mathrm{OS}, \mathrm{P}=0.036$ ) remained as independent predictors for poor PFS and OS (Table 3).

\section{In PKM2 high expression cohort alone, treat- ment employed EPI or EPI plus 5-Fu displayed a good clinical outcome}

Next we focused on the cohort of patients with PKM2 high expression. All the patients were separated into three groups according to chemotherapy regimen treatments. Group 1, non-EPI/5-Fu chemotherapy (regimens without EPI and 5-Fu); Group 2, EPI-based chemotherapy (without 5-Fu); Group 3, EPI plus 5-Fu based chemotherapy were defined as shown alone. The reason of this grouping method was explained in the method of Neoadjuvant chemotherapy verification cases. We found that patients in Group 2 (PFS, $\chi 2=8.788, P=0.003$, OS, $\chi 2=9.050, P=0.003$ ) and those in Group 3 (PFS, $\chi 2=6.177, \mathrm{P}=0.013$, OS, $\chi 2=8.326, P=0.004)$ had better survival than patients in Group 1, respectively (Fig. 5A, 5B). In contrast, there was no significant difference in PFS or OS among the three groups in the cohort of PKM2 low expression patients $(P>0.05)$ (Fig. 5C, 5D). 
Table 1. Comparison of the patients' characteristics and clinicopathological features between low and high PKM2 expression groups

\begin{tabular}{|c|c|c|c|c|c|}
\hline \multirow{3}{*}{$\begin{array}{l}\text { Clinicopathological } \\
\text { variables }\end{array}$} & \multicolumn{5}{|c|}{ PKM2 expression } \\
\hline & \multicolumn{2}{|l|}{ Low } & \multicolumn{2}{|c|}{ High } & \multirow[t]{2}{*}{$P$ value } \\
\hline & No. & $\%$ & No. & $\%$ & \\
\hline \multicolumn{6}{|l|}{ Age(years) } \\
\hline$<50$ & 58 & 40.6 & 71 & 46.4 & 0.311 \\
\hline$\geq 50$ & 85 & 59.4 & 82 & 53.6 & \\
\hline \multicolumn{6}{|l|}{ Menopausal status } \\
\hline pre & 57 & 39.9 & 67 & 43.8 & 0.493 \\
\hline post & 86 & 60.1 & 86 & 56.2 & \\
\hline \multicolumn{6}{|c|}{ Family history of cancer } \\
\hline yes & 41 & 31.1 & 44 & 31.9 & 0.884 \\
\hline no & 91 & 68.9 & 94 & 68.1 & \\
\hline \multicolumn{6}{|l|}{ Tumor size ( $T$ stage) } \\
\hline $\mathrm{T} 1$ & 63 & 45 & 87 & 58.8 & $0.008^{*}$ \\
\hline $\mathrm{T} 2$ & 59 & 42.1 & 55 & 37.2 & \\
\hline T3 & 18 & 12.9 & 6 & 4 & \\
\hline \multicolumn{6}{|l|}{ Lymph node stage } \\
\hline No & 82 & 58.1 & 66 & 43.7 & $0.047^{*}$ \\
\hline N1 & 31 & 22.0 & 37 & 24.5 & \\
\hline N2 & 11 & 7.8 & 24 & 15.9 & \\
\hline N3 & 17 & 12.1 & 24 & 15.9 & \\
\hline \multicolumn{6}{|l|}{ Histological grade } \\
\hline I & 9 & 6.3 & 10 & 6.6 & 0.886 \\
\hline II & 106 & 74.7 & 109 & 72.2 & \\
\hline III & 27 & 19 & 32 & 21.2 & \\
\hline \multicolumn{6}{|l|}{ ER status } \\
\hline NEG & 47 & 33.8 & 51 & 33.3 & 0.931 \\
\hline POS & 92 & 66.2 & 102 & 66.7 & \\
\hline \multicolumn{6}{|l|}{ PR status } \\
\hline NEG & 66 & 47.5 & 66 & 43.1 & 0.456 \\
\hline POS & 73 & 52.5 & 87 & 56.9 & \\
\hline \multicolumn{6}{|l|}{ HER2 status } \\
\hline NEG & 123 & 88.5 & 135 & 88.2 & 0.946 \\
\hline POS & 16 & 11.5 & 18 & 11.8 & \\
\hline \multicolumn{6}{|l|}{ Ki67 status } \\
\hline$<20$ & 57 & 41.9 & 59 & 39.3 & 0.657 \\
\hline$\geq 20$ & 79 & 58.1 & 91 & 60.7 & \\
\hline \multicolumn{6}{|l|}{ Molecular subtypes } \\
\hline Luminal A & 55 & 40.4 & 58 & 38.7 & 0.815 \\
\hline Luminal B & 35 & 25.7 & 46 & 30.7 & \\
\hline HER2 enriched & 13 & 9.6 & 14 & 9.3 & \\
\hline Basal like & 33 & 24.3 & 32 & 21.3 & \\
\hline \multicolumn{6}{|l|}{ Clinical staging } \\
\hline I & 38 & 27.5 & 37 & 25.2 & 0.255 \\
\hline II & 68 & 49.3 & 62 & 42.2 & \\
\hline III & 31 & 22.5 & 44 & 29.9 & \\
\hline IV & 1 & 0.7 & 4 & 2.7 & \\
\hline
\end{tabular}

Lymph node stage: N0, indicates no lymph node metastasis; N1, 1-3 lymph node metastasis; N2, 4-9 lymph node metastasis; N3, $\geq 10$ lymph node metastasis; ER: estrogen receptor; PR: progesterone receptor; HER-2: human epidermal growth factor receptor 2; NEG: negative expression; POS: positive expression; Analysis was limited to patients for whom data were available; *indicates $\mathrm{P}<0.05$.

Table 2. Breast cancer patients' chemosensitivity to each anticancer drug based on the CD-DST

\begin{tabular}{|c|c|c|c|c|c|c|}
\hline \multirow{2}{*}{$\begin{array}{l}\text { Anticancer } \\
\text { drug }\end{array}$} & \multicolumn{2}{|c|}{ Non-sensitive } & \multicolumn{2}{|c|}{ Sensitive } & \multirow{2}{*}{$\begin{array}{l}\text { Concentration } \\
(\mu \mathrm{g} / \mathrm{ml})\end{array}$} & \multirow{2}{*}{$\begin{array}{l}\text { Exposure } \\
\text { time (hr) }\end{array}$} \\
\hline & No. & $\%$ & No. & $\%$ & & \\
\hline EPI & 170 & 62.3 & 103 & 37.7 & 0.3 & 24 \\
\hline CDDP & 187 & 68.5 & 86 & 31.5 & 2 & 24 \\
\hline NVB & 207 & 73.7 & 74 & 26.3 & 10 & 24 \\
\hline PAC & 81 & 51.3 & 77 & 48.7 & 10 & 24 \\
\hline 5-Fu & 108 & 73 & 40 & 27 & 10 & 24 \\
\hline
\end{tabular}

Cases with $\mathrm{T} / \mathrm{C}$ ratio of $\leq 50 \%$ were defined as in vitro sensitive group, cases with

$\mathrm{T} / \mathrm{C}$ ratio of $>50 \%$ were defined as in vitro non-sensitive group.
Univariate and multivariate analysis confirmed that chemotherapy employed EPI or EPI plus 5-Fu was associated with a longer survival in patients with high PKM2 expression $(\mathrm{P}<0.05)$. Meanwhile, lymph node status was also an independent prognostic factor for PFS and OS in PKM2 high cohort $(\mathrm{P}<0.05)$. In addition, the univariate analysis indicated tumor size was a poor indicator in PFS and OS in PKM2 high expression cohort $(\mathrm{P}<0.05)$. (Table. 4)

\section{Discussion}

In previous studies, PKM2 has been indicated to be notably up-regulated in different types of cancers and responsible for their poor prognosis. Wen et. al claimed PKM2 as a hallmark of cancer whose expression levels were correlated with disease-free and overall survival in a cohort of 490 hepatocellular carcinoma patients [22]. Zhang et al. demonstrated that high PKM2 status was associated with a decreased overall life time in esophageal squamous cell cancer [23]. At the same time, PKM2 was also reported to have high expression in breast cancers [24, 25]. In this study we found that in a group of 296 breast cancer patients, PKM2 was universally expressed and closely correlated with malignant clinical characteristic such as lymph node stage. Furthermore, our result showed that high levels of PKM2 expression served as an independent risk factor indicating unfavorable PFS and OS in breast cancer patients. This may indicate that PKM2 status could be helpful to evaluate patients who should be under close surveillance for tumor recurrence and metastasis. In view of this, it is urgent to find effective chemotherapy drugs to overcome the poor prognosis of high PKM2 status in breast cancer.

In the present research, we studied the relationship between PKM2 status and the sensitivity of primary breast cancer cells to chemotherapy drugs. The sensitivity of primary cancer cells to chemotherapy drugs was examined by CD-DST. CD-DST has been shown to accurately reflect individual drug sensitivity in vitro in breast cancer. Tozuka et al found a subgroup of ER-positive and HER2-negative breast cancer patients whom displaying good responses to chemotherapy based on CD-DST and the status of Ki67 [18]. Takamura et al surveyed the correlation between CD-DST results and clinical chemotherapeutic responses they claimed that CD-DST was capable of predicting a response to cyclophosphamide and epirubicin treatment or doxorubicin treatment with excellent accurateness in patients with breast cancer [17]. In our research, we employed 296 surgical resection specimens of breast cancer to perform CD-DST. Each patient's specimen received 4 types of anticancer drugs randomly, and the sensitivity rates to EPI and $5-\mathrm{Fu}$ is $33.7 \%$ (103/283), $27 \%$ (40/148) respectively. 
We found the patients with the better chemosensitivity to EPI and 5-Fu in vitro had the higher PKM2 ex-

pression status, and this result was statistically significant.

Table 3. Univariate and multivariate analyses for PFS and OS in breast cancer patients

\begin{tabular}{|c|c|c|c|c|c|c|}
\hline \multirow[t]{2}{*}{ Variables } & \multicolumn{3}{|c|}{ PFS } & \multicolumn{3}{|c|}{ OS } \\
\hline & $\overline{\text { HR }}$ & $95 \% \mathrm{CI}$ & $P$ value & HR & $95 \% \mathrm{CI}$ & $P$ value \\
\hline Age(<50 vs. $\geq 50)$ (years) & 0.819 & $0.478-1.403$ & 0.467 & 0.922 & $0.507-1.677$ & 0.790 \\
\hline Tumor size $(\leq 2$ vs. $>2)(\mathrm{cm})$ & 2.167 & $1.115-4.209$ & $0.022^{*}$ & 2.092 & $1.003-4.362$ & $0.049^{*}$ \\
\hline Lymph node (negative vs. positive) & 2.15 & $1.240-3.730$ & $0.006^{*}$ & 2.108 & $1.144-3.884$ & $0.017^{*}$ \\
\hline Histological grade ( I vs. II vs. III) & 1.319 & $0.775-2.244$ & 0.308 & 1.603 & $0.904-2.842$ & 0.107 \\
\hline HER2 status (negative vs. positive) & 2.288 & $1.149-4.559$ & $0.019^{*}$ & 3.121 & $1.538-6.335$ & $0.002^{*}$ \\
\hline Ki67 status $(<20 \%$ vs. $\geq 20 \%)$ & 2.726 & $1.400-5.305$ & $0.003^{*}$ & 4.491 & $1.895-10.64$ & $0.001^{*}$ \\
\hline PKM2 status (low vs. high) & 2.351 & $1.308-4.227$ & $0.004^{*}$ & 2.347 & $1.224-4.500$ & $0.010^{*}$ \\
\hline \multicolumn{7}{|l|}{ Multivariate analysis } \\
\hline Tumor size $(\leq 2$ vs. $>2)(\mathrm{cm})$ & 1.700 & $0.848-3.405$ & 0.135 & 1.545 & $0.850-3.862$ & 0.271 \\
\hline Lymph node (negative vs. positive) & 1.838 & $1.013-3.336$ & $0.045^{*}$ & 2.136 & $1.081-4.219$ & $0.029^{*}$ \\
\hline HER2 status (negative vs. positive) & 1.676 & $0.821-3.423$ & 0.156 & 1.997 & $0.963-4.144$ & 0.063 \\
\hline Ki67 status $(<20 \%$ vs. $\geq 20 \%)$ & 2.522 & $1.285-4.948$ & $0.007^{*}$ & 4.053 & $1.696-9.690$ & $0.002^{*}$ \\
\hline PKM2 status (low vs. high) & 2.135 & $1.161-3.927$ & $0.015^{*}$ & 1.910 & $0.984-3.708$ & $0.036^{*}$ \\
\hline
\end{tabular}

*indicates $\mathrm{P}<0.05$. HR, hazard ratio; $\mathrm{CI}$, confidence interval.

Table 4. Univariate and multivariate analyses for PFS and OS in PKM2 high expression cohort

\begin{tabular}{|c|c|c|c|c|c|c|}
\hline \multirow[t]{2}{*}{ Variables } & \multicolumn{3}{|c|}{ PFS } & \multicolumn{3}{|c|}{ OS } \\
\hline & HR & $95 \% \mathrm{CI}$ & $P$ value & HR & $95 \% \mathrm{CI}$ & $P$ value \\
\hline \multicolumn{7}{|l|}{ Univariate analysis } \\
\hline Age(<50 vs. $\geq 50)$ (years) & 0.611 & $0.317-1.179$ & 0.142 & 0.685 & $0.335-1.398$ & 0.298 \\
\hline Tumor size $(\leq 2$ vs. $>2)(\mathrm{cm})$ & 2.563 & $1.128-5.822$ & $0.025^{*}$ & 3.018 & $1.161-7.840$ & $0.023^{*}$ \\
\hline Lymph node (N0 vs. N1 vs. N2+N3) & 1.701 & $1.195-2.420$ & $0.003^{*}$ & 1.721 & $1.169-2.534$ & $0.006^{*}$ \\
\hline Histological grade ( I vs. II vs. III) & 1.066 & $0.578-1.965$ & 0.837 & 1.068 & $0.561-2.033$ & 0.841 \\
\hline \multicolumn{7}{|l|}{ Clinical treatments } \\
\hline $\begin{array}{l}\text { Chemotherapy (non-EPI/5-Fu vs. EPI-based + EPI } \\
\text { plus 5-Fu) }\end{array}$ & 0.348 & $0.180-0.674$ & $0.002^{*}$ & 0.302 & $0.149-0.614$ & $0.001^{*}$ \\
\hline Endocrine therapy (no vs. yes) & 0.938 & $0.596-2.532$ & 0.576 & 1.137 & $0.510-2.531$ & 0.754 \\
\hline Bio-therapy (no vs. yes) & 0.607 & $0.321-1.149$ & 0.125 & 0.534 & $0.266-1.075$ & 0.079 \\
\hline Radio-therapy (no vs. yes) & 1.384 & $0.733-2.614$ & 0.317 & 1.482 & $0.740-2.969$ & 0.267 \\
\hline \multicolumn{7}{|l|}{ Multivariate analysis } \\
\hline Tumor size $(\leq 2$ vs. $>2)(\mathrm{cm})$ & 1.659 & $0.695-3.960$ & 0.254 & 1.848 & $0.668-5.110$ & 0.237 \\
\hline Lymph node (N0 vs. N1 vs. N2+N3) & 1.577 & $1.087-2.288$ & $0.016^{*}$ & 1.555 & $1.034-2.338$ & $0.034^{*}$ \\
\hline Chemotherapy & 0.355 & $0.181-0.698$ & $0.003^{*}$ & 0.322 & $0.157-0.663$ & $0.002^{*}$ \\
\hline
\end{tabular}

*indicates $\mathrm{P}<0.05$

A

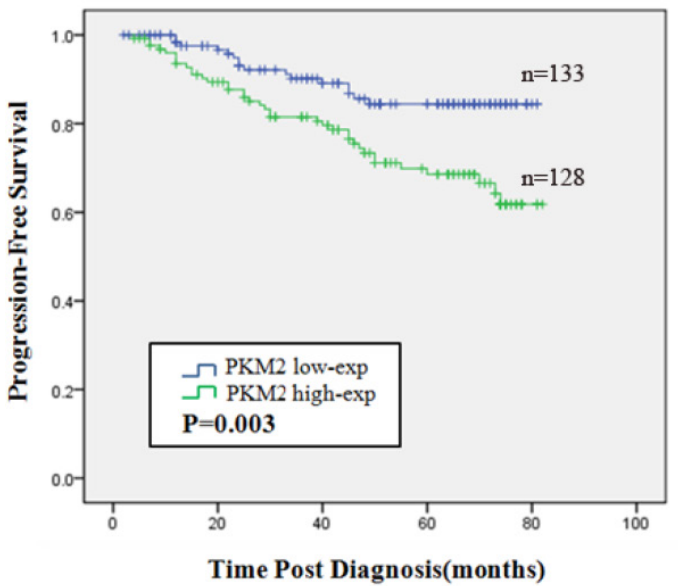

B

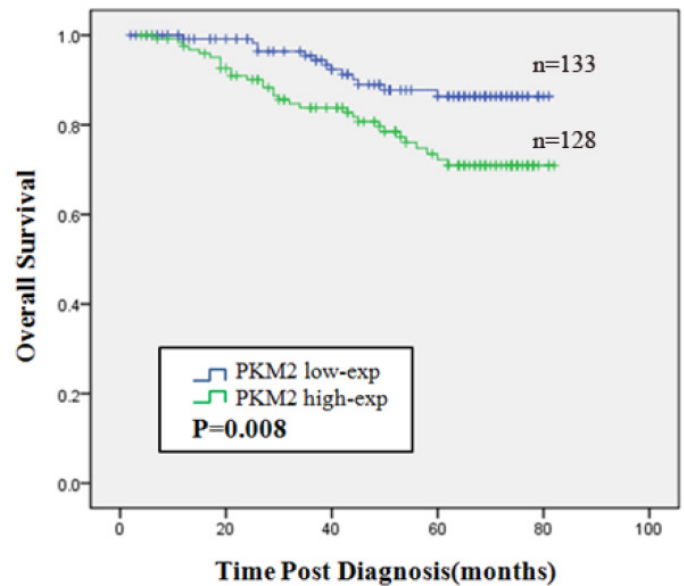

Fig 4. Prognostic significance of PKM2 expression in breast cancer. Kaplan-Meier analysis of progression-free survival (PFS) (A) and overall survival (OS) (B) were stratified by the cutoff value for dividing into low and high PKM2 expression groups. Blue line represented patients with low expression of PKM2 and green line indicated patients with high expression of PKM2. $P$ values were computed by the log-rank test. 
A

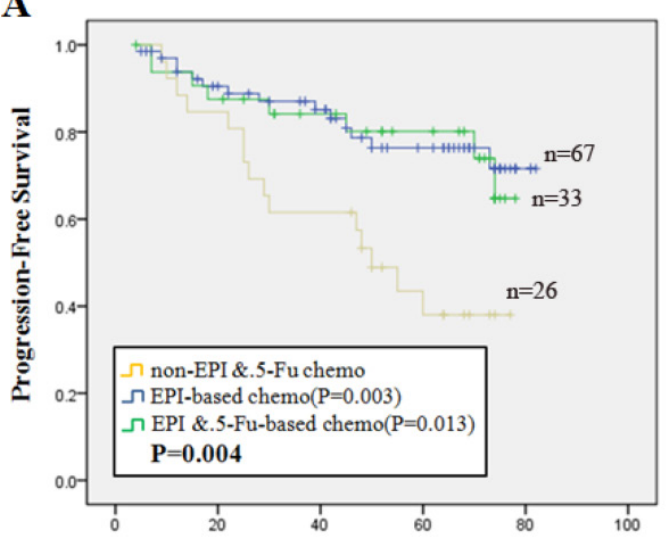

B

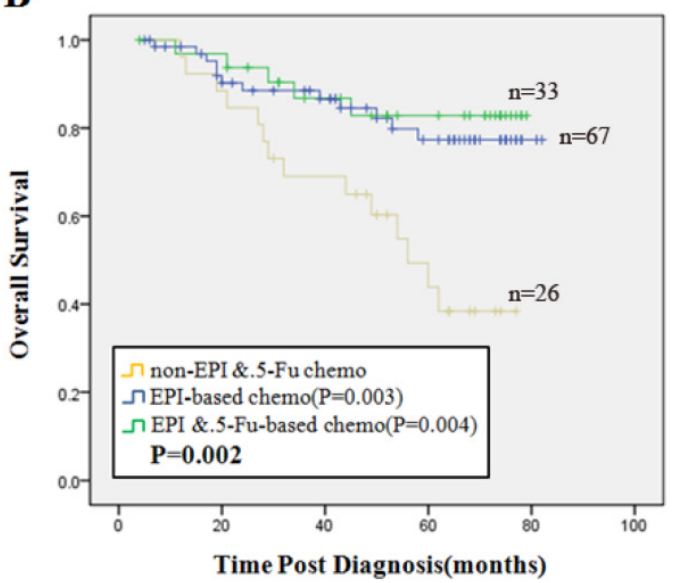

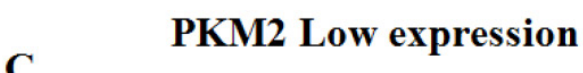

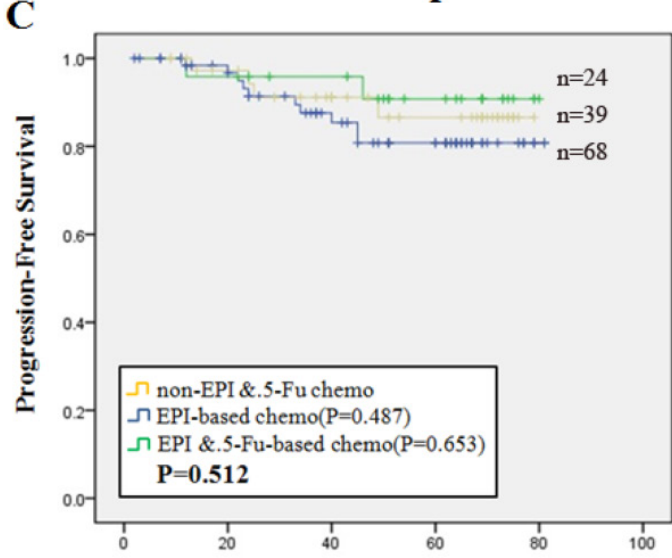

D

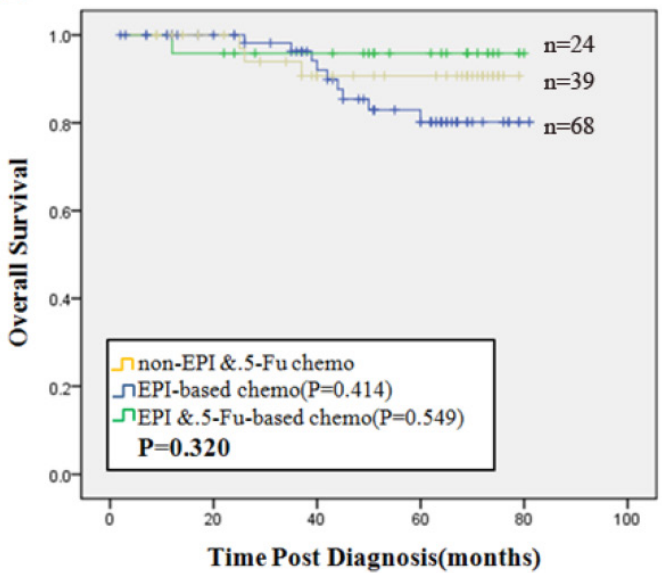

Fig 5. Predictive efficacy of PKM2 status among three subsets of treatment. Kaplan-Meier analysis of PFS (A) and OS (B) in patients with high PKM2 status illustrated significant differences among three chemotherapy groups (PFS, $\mathrm{P}=0.004, \mathrm{OS}, \mathrm{P}=0.002$ ). $\mathrm{PFS}(\mathrm{C})$ and $\mathrm{OS}(\mathrm{D})$ in patients with low $\mathrm{PKM} 2$ status displayed no significance among the three groups ( $P F S, P=0.512$, OS, $P=0.320$ ). Blue line stands for EPI-based (without 5 -Fu) chemotherapy, green line stands for EPI plus 5 -Fu based chemotherapy, and yellow line stands for non-EPI/5-Fu chemotherapy. $P$ values were computed by the log-rank test.

Then we used neoadjuvant chemotherapy cases to confirm the conclusion. There were only $3-26 \%$ of breast cancer patients who got $\mathrm{pCR}$ after neoadjuvant chemotherapy, and pCR could be a good indicator of survival time [26]. Even though the greater part of neoadjuvant chemotherapy cases did not realize $\mathrm{pCR}$, cancer cells could still make a response to chemotherapy in a pathologically important consequence which could be seen by microscopy [27]. In this study, PKM2 expression levels were significant correlated with pathological response to EPI-based and EPI plus 5-Fu chemotherapy. The better pathological response to EPI and 5-Fu, the higher PKM2 expression levels were seen in patients. From this result, we obtained in vivo evidence to verify that high PKM2 expression was linked to patients' individual chemosensitivity to EPI and 5-Fu.

In high PKM2 expression cohort, we found that patients who received EPI-based or EPI plus 5-Fu chemotherapy had a favorable PFS and OS than non-EPI/5-Fu treated patients, respectively. From the perspective of prognosis this result confirmed the association between PKM2 expression levels and chemosensitivity to EPI and 5-Fu in breast cancer. While the role of PKM2 as a reporter protein to reflect cancer cells chemosensitivity is not yet fully explored and seems to be controversial. Wang and his colleagues reported inhibition of PKM2 sensitized triple-negative breast cancer cell lines to doxorubicin, and the efficacy of combination treatment displayed better than doxorubicin alone [28]. Another study showed in 5-Fu-resistant cells overexpression of miR-122 could inhibit the expression of PKM2 both in vivo and in vitro which was followed by overcoming 5-Fu resistance [29]. And inhibited PKM2 activity in breast adenocarcinoma MBA-MD-231 cells by a small-molecule product-shikonin could sensitize the therapeutic efficacy of paclitaxel [30]. These results indicated PKM2 was associated with less sensitivity to anticancer drugs treatment. In contrast, other studies showed opposite results. Martinez et al. reported in oxaliplatin-resistant cell line the expression of PKM2 was decreased by proteomics studies, meanwhile in colorectal cancer patients who got better response rate 
in oxaliplatin-treated had the higher mRNA expression of PKM2 [31]. Yoo et al showed that the PKM2 protein levels and activity decreased in cisplatin-resistant human gastric carcinoma cell lines compared with the parental cell lines. Moreover, employing antisense oligonucleotides to inhibit PKM2 expression could decline tumors sensitivity to cisplatin [32]. Li et al further claimed PKM2 inhibition enhanced ovarian cancer cells chemoresistance to cisplatin [33]. In Papadaki's research, two different outcomes were showed at the same time. First, low mRNA levels of PKM2 were related to a favorable prognosis of NSCLC patients who received platinum-based treatment. Second, they found that $80 \%$ of the patients whose primary tumors had lower PKM2 mRNA levels did not respond to first-line chemotherapy treatment with oxaliplatin/5Fu regimen [34]. This discrepancy might be due to the fact that PKM2 as a regulator is involved in metabolic pathways of different anticancer drugs through multiple mechanisms. Further researches are extremely warranted to explain the molecular mechanisms of the consequence.

It should be mentioned that there were no apparent differences in efficacy between EPI-based (without 5-Fu) and EPI plus 5-Fu based treatment in PKM2 high expression group. We thought there were three possible explanations for this result. Firstly, the anticancer mechanism of EPI and 5-Fu are both inhibitors of DNA and RNA synthesis; hence, it is reasonable to get the same efficacy when the drugs act on the same effect. Secondly, breast cancer patients treated with 5-Fu are usually considered to be of high malignant of clinical assessment, the combination of EPI and 5-Fu may counterpoise this kind of cases deviation in final efficacy. Thirdly, combination therapy with other anticancer drugs such as cyclophosphamide might lead to some additional influence.

In addition, it was interesting that the status of PKM2 was negatively associated with tumor size analyzed in the present study. There were more T1 stage and less T3 stage tumors in high PKM2 expression group. However, tumor size was a poor predictor of PFS and OS as indicated in the survival analysis both in overall patients and in high PKM2 patients. These results revealed high PKM2 breast tumors have a more malignant characteristic compared with low PKM2 breast tumors even in a smaller tumor size.

\section{Conclusion}

Taken together, PKM2 status may be a predictor for evaluating prognosis of invasive breast carcinoma. This is the first report to show a relationship between PKM2 expression and patient's individual sensitivity to EPI and 5-Fu in breast cancer. Our data suggests that patients with high PKM2 status of primary tumors should be considered for using of EPI based or EPI plus 5-Fu chemotherapy in order to obtain a better prognosis.

\section{Abbreviations}

PKM2: Pyruvate kinase M2; CD-DST: collagen gel droplet embedded culture-drug sensitivity test; EPI: epirubicin; 5-Fu: 5-fluorouracil; PFS: progression free survival; OS: overall survival; CDDP: Cisplatin; NVB: Vinorelbine; PAC: Paclitaxel; pCR: pathological complete response; pPR: pathological partial response; pNR: no pathological response; HR: hazard ratio; $\mathrm{CI}$ : confidence interval.

\section{Acknowledgments}

Research was supported by grants from the National Natural Science Foundation of China (30930038, 81272359,81202101 ) and Initial Funding for Doctors of Tianjin Medical University Cancer Institute and Hospital (B1215). We are very grateful to Dr. Gerald J. Mizejewski ( Ph.D, Wadsworth Center, New York State Department of Health, NY 12201, United States) for proofreading the manuscript and Dr. Ping Du ( M.D, Ph.D, Biostatistician, Penn State University Medical College) for editing the statistical part of the manuscript.

\section{Competing Interests}

The authors have declared that no competing interest exists.

\section{References}

1. Jemal A, Bray F, Center MM, Ferlay J, Ward E, Forman D. Global cancer statistics. CA Cancer J Clin. 2011; 61(2):69-90.

2. Naitoh H, Yamamoto H, Murata S, Kobayashi H, Inoue K, Tani T. Stratified phase II trial to establish the usefulness of the collagen gel droplet embedded culture-drug sensitivity test (CD-DST) for advanced gastric cancer. Gastric Cancer. 2014; 17(4):630-7.

3. Yabushita H, Ohnishi M, Komiyama M et al. Usefulness of collagen gel droplet embedded culture drug sensitivity testing in ovarian cancer. Oncol Rep. 2004; 12(2): 307-311.

4. Tamura Y, Kobayashi H, Taguchi T, Motoyama K, Inaji H and Noguchi S. Prediction of chemotherapeutic response by collagen gel droplet embedded culture-drug sensitivity test in human breast cancers. Int j Cancer. 2002; 98(3): 450-455

5. Mekata E, Sonoda H, Shimizu T et al. Clinical predictive value of in vitro anticancer drug sensitivity test for the therapeutic effect of adjuvant chemotherapy in patients with stage II-III colorectal cancer. Mol Clin Oncol. 2013; 1(4):763-767.

6. Cairns RA, Harris IS, and Mak TW. Regulation of cancer cell metabolism. Nat Rev Cancer. 2011; 11(2): 85-95.

7. Vander Heiden MG, Cantley LC, Thompson CB. Understanding the Warburg Effect: The Metabolic Requirements of Cell Proliferation. Science. 2009; 324(5930):1029-33.

8. Christofk HR, Vander Heiden MG, Wu N, Asara JM, Cantley LC. Pyruvate kinase M2 is a phosphotyrosine-binding protein. Nature. 2008; 452(7184):181-186.

9. Christofk HR, Vander Heiden MG, Harris MH et al. The M2 splice isoform of pyruvate kinase is important for cancer metabolism and tumour growth. Nature. 2008; 452(7184): 230-233

10. Mazurek S. Pyruvate kinase type M2: a key regulator of the metabolic budget system in tumor cells. Int J Biochem Cell Biol. 2011; 43(7):969-80.

11. Wang HJ, Hsieh YJ, Cheng WC et al. JMJD5 regulates PKM2 nuclear translocation and reprograms HIF-1a-mediated glucose metabolism. Proc Natl Acad Sci U S A. 2014; 111(1):279-84 
12. Warner SL, Carpenter KJ, Bearss DJ. Activators of PKM2 in cancer metabolism. Future Med Chem. 2014; 6(10):1167-78.

13. Fukuda S, Miyata H, Miyazaki Y et al. Pyruvate Kinase M2 Modulates Esophageal Squamous Cell Carcinoma Chemotherapy Response by Regulating the Pentose Phosphate Pathway. Ann Surg Oncol. 2015; [Epub ahead of print].

14. Meng MB, Wang $\mathrm{HH}$, Guo $\mathrm{WH}$ et al. Targeting pyruvate kinase $\mathrm{M} 2$ contributes to radiosensitivity of non-small cell lung cancer cells in vitro and in vivo. Cancer Lett. 2015; 356(2 Pt B):985-93.

15. Elston CW, Ellis IO. Pathological prognostic factors in breast cancer. I. The value of histological grade in breast cancer: experience from a large study with long-term follow-up. Histopathology. 2002; 41(3A):154-61.

16. Kobayashi $\mathrm{H}$, Tanisaka $\mathrm{K}$, Doi $\mathrm{O}$ et al. An in vitro chemosensitivity test for solid human tumors using collagen gel droplet embedded cultures. Int J Oncol. 1997; 11(3): 449-55.

17. Takamura Y, Kobayashi H, Taguchi T, Motomura K, Inaji H, Noguchi S. Prediction of chemotherapeutic response by collagen gel droplet embedded culture-drug sensitivity test in human breast cancers. Int J Cancer. 2002; 98(3):450-5.

18. Tozuka K, Horiguchi J, Takata D et al. Collagen gel droplet-embedded culture-drug sensitivity test and Ki67 expression in estrogen receptor-positiveand HER2-negative breast cancer. Mol Clin Oncol. 2013; 1(1):93-99.

19. Cheang MC, Chia SK, Voduc D et al. Ki67 index, HER2 status, and prognosis of patients with luminal B breast cancer. J Natl Cancer Inst. 2009; 101(10):736-750.

20. Goldhirsch A, Winer EP, Coates AS, Gelber RD, Piccart-Gebhart M, Thürlimann B, Senn HJ. Personalizing the treatment of women with early breast cancer: highlights of the St Gallen International Expert Consensus on the Primary Therapy of Early Breast Cancer 2013. Ann Oncol. 2013 Sep;24(9):2206-23.

21. Adams $S$, Chakravarthy $A B$, Donach $M$ et al. Preoperative concurrent paclitaxel- radiation in locally advanced breast cancer: pathologic response correlates with five-year overall survival. Breast Cancer Res Treat. 2010; 124(3):723-32

22. $\mathrm{Hu} \mathrm{W}, \mathrm{Lu} \mathrm{SX}, \mathrm{Li} \mathrm{M}$ et al. Pyruvate kinase M2 prevents apoptosis via modulating Bim stability and associates with poor outcome in hepatocellular carcinoma. Oncotarget. 2015; 6(9):6570-83.

23. Zhang $X, H e C$, Chen $B$ et al. Nuclear PKM2 expression predicts poor prognosis in patients with esophageal squamous cell carcinoma. Pathol Res Pract. 2013; 209(8):510-515.

24. Ishikawa M, Inoue $\mathrm{T}$, Shirai $\mathrm{T}$ et al. Simultaneous expression of cancer stem cell-like properties and cancer-associated fibroblast-like properties in a primary culture of breast cancer cells. Cancers (Basel).2014; 6(3):1570-8.

25. Shashni B, Sakharkar KR, Nagasaki Y, Sakharkar MK. Glycolytic enzymes PGK1 and PKM2 as novel transcriptional targets of PPARy in breast cancer pathophysiology. J Drug Target.2013; 21(2):161-74.

26. Montagna E, Bagnardi V, Rotmensz $\mathrm{N}$ et al. Pathological complete response after preoperative systemic therapy and outcome: relevance of clinical and biologic baseline features. Breast Cancer Res Treat. 2010; 124(3): 689-99.

27. Balmativola $\mathrm{D}$, Marchiò $\mathrm{C}$, Maule $\mathrm{M}$ et al. Pathological non-response to chemotherapy in a neoadjuvant setting of breast cancer: an inter-institutional study. Breast Cancer Res Treat. 2014; 148(3):511-23.

28. Wang F, Yang Y. Inhibition of PKM2 sensitizes triple-negative breast cancer cells to doxorubicin. Biochem Biophys Res Commun. 2014; 454(3):465-470.

29. He J, Xie G, Tong J et al. Overexpression of microRNA-122 re-sensitizes 5-FUresistant colon cancer cells to 5-FU through the inhibition of PKM2 in vitro and in vivo. Cell Biochem Biophys. 2014; 70(2):1343-50.

30. Li W, Liu J, Jackson K, Shi R, Zhao Y. Sensitizing the therapeutic efficacy of taxol with shikonin in human breast cancer cells. PLoS One. 2014; 9(4):e94079.

31. Martinez-Balibrea E, Plasencia C, Gines A. A proteomic approach links decreased pyruvate kinase M2 expression to oxaliplatin resistance in patients with colorectal cancer and in human cell lines. Mol Cancer Ther. 2009; 8(4): 771-778.

32. Yoo BC, Ku JL, Hong SH et al. Decreased pyruvate kinase M2 activity linked to cisplatin resistance in human gastric carcinoma cell lines. Int J Cancer. 2004; 108(4): 532-539.

33. Li SL, Ye F, Cai WJ et al. Quantitative proteome analysis of multidrug resistance in human ovarian cancer cell line. J Cell Biochem. 2010; 109(4):625-33.

34. Papadaki C, Sfakianaki M, Lagoudaki E et al. PKM2 as a biomarker for chemosensitivity to front-line platinum-based chemotherapy in patients with metastatic non-small-cell lung cancer. British Journal of Cancer. 2014; 111(9): $1757-1764$. 